\title{
La Société Suisse d'Ethique Biomédicale - 20 ans de dialogue entre médecine et société
}

Samia Hurst

Prof., Présidente SSEB

Médecin et bioéthicienne

La médecine est une activité humaine au sens le plus fondamental du terme. Elle touche à l'intimité des personnes malades; les valeurs qui s'y déploient nous mettent face aux tensions, et parfois aux contradictions, de notre vie morale. Les difficultés éthiques y sont fréquentes et cela ne doit pas nous étonner: c'est là le résultat de la conjonction d'un terrain particulièrement difficile au contact des limites humaines, et de professionnels auxquels l'éthique importe. Des personnes raisonnables peuvent être en désaccord dans les dilemmes qui surviennent dans la pratique clinique.

\section{Notre point commun n'est pas une formation identique, \\ mais un intérêt commun pour les difficultés éthiques soulevées dans la médecine et les sciences du vivant}

Correspondance: Prof. Samia Hurst Institut d'éthique biomédicale 1 , rue Michel Servet CH-1211 Genève 4

samia.hurst@unige.ch

www.bioethics.ch
Ces difficultés constituent une part de la raison d'être de l'éthique biomédicale. Constituée dans une rencontre entre des cliniciens confrontés à des difficultés éthiques profondes, et les praticiens de la jeune discipline théorique qu'était alors la bioéthique, elle a eu des débuts difficiles. En 1973, Daniel Callahan, un de ses pionniers outre-Atlantique, avoue carrément: «Je résistais, avec une pure panique, à l'idée de participer avec les médecins dans leurs décisions. Moi? Je préférais nettement la sécurité des questions profondes que je poussais vers eux. Mais je réalisais aussi en étant confronté à de véritables situations - et c'est là mon excuse - qu'il n'y avait rien dans ma formation philosophique pour me préparer à prendre une décision éthique claire à une heure donnée d'un après-midi précisé. J'avais été formé comme il faut dans une splendide tradition d'érudition et de pensée soigneuse qui laisse au moins un ou deux millénaires pour résoudre un problème.» [1]. Cette rencontre, malgré ces difficultés, se passa bien, et fut utile aux uns et aux autres. Des philosophes se formèrent à la réalité des soins, des médecins prirent le temps d'apprendre sérieusement la théorie morale. Des consultants d'éthique issus de plusieurs disciplines furent accueillis dans les hôpitaux, où ils furent appréciés, et leur regard enrichit la pratique clinique [2].

Fondée il y a tout juste 20 ans en 1989, la Société Suisse d'Ethique Biomédicale (SSEB) est issue des mêmes origines. Elle a pour but d'offrir un forum au sein duquel les difficultés soulevées par les progrès de la médecine, mais également par la pratique quotidienne, peuvent être discutés librement et des solutions trouvées dans un échange interdisciplinaire respectueux. Ses membres sont médecins, philosophes, infirmiers, juristes, théologiens, éthiciens, entre autres. Toute personne intéressée peut en devenir membre.

Notre point commun n'est pas une formation identique, mais un intérêt commun pour les difficultés éthiques soulevées dans la médecine et les sciences du vivant. L'existence de la SSEB est fondée sur le souci de contribuer à une délibération ouverte, interdisciplinaire, et démocratique de ces enjeux.

Dans ce but, la SSEB

- aborde des questions d'éthique fondamentale au cours de séminaires et organise des colloques sur des thèmes éthiques controversés.

- publie Bioethica Forum, une revue interdisciplinaire de bioéthique trilingue (all/fr/ang) à politique éditoriale [3]; Folia Bioethica, une série d'ouvrages brefs sur des sujets d'actualité; la newsletter SGBEbulletinSSEB.

- réunit une fois par an lors d'un symposium les chercheurs en bioéthique de Suisse.

- participe à la planification et l'organisation de cours de base et de formation continue pour les membres de commissions d'éthique de la recherche sur l'être humain.

\section{Références}

1 Callahan D. Bioethics as a discipline. In: Jecker NS, Jonsen AR, Pearlman RA, editors. Bioethics. Boston, London, Singapore: Jones and Bartlett; 1997

2 Hurst SA. La consultation d'éthique: à quoi ça sert et comment s'en servir? Revue Médicale Suisse. 2006;80:2195-9.

3 www.bioethica-forum.ch

\section{Fête d'anniversaire}

La Société Suisse d'Ethique Biomédicale fête ses 20 ans le 4 décembre 2009 lors d'un symposium à l'Hôpital de I'lle de Berne autour du thème «Le Futur de la Bioéthique». C'est là une bonne occasion de faire connaissance avec un domaine tourné vers des questions dont l'importance grandit au sein de la pratique clinique et de notre système de santé.

Programme: www.sgbe-sseb.ch; Inscriptions auprès de: info@bioethics.ch 